\title{
Scaling of Relativistic Shear Flows with the Bulk Lorentz Factor
}

\author{
Edison Liang ${ }^{1}$, Wen Fu ${ }^{1}(1)$, Markus Böttcher ${ }^{2}$ (1) , and Parisa Roustazadeh ${ }^{2}$ \\ ${ }^{1}$ Rice University, Houston, TX 77005, USA \\ 2 North-West University, Potchefstroom, 2520, South Africa \\ Received 2017 October 22; revised 2017 December 24; accepted 2018 January 8; published 2018 February 20
}

\begin{abstract}
We compare Particle-in-cell simulation results of relativistic electron-ion shear flows with different bulk Lorentz factors, and discuss their implications for spine-sheath models of blazar versus gamma-ray burst (GRB) jets. Specifically, we find that most properties of the shear boundary layer scale with the bulk Lorentz factor: the lower the Lorentz factor, the thinner the boundary layer, and the weaker the self-generated fields. Similarly, the energized electron spectrum peaks at an energy near the ion drift energy, which increases with bulk Lorentz factor, and the beaming of the accelerated electrons along the shear interface gets narrower with increasing Lorentz factor. This predicts a strong correlation between emitted photon energy, angular beaming, and temporal variability with the bulk Lorentz factor. Observationally, we expect systematic differences between the high-energy emissions of blazars and GRB jets.
\end{abstract}

Key words: galaxies: active - gamma-ray burst: general - magnetic fields - physical data and processes

\section{Introduction}

Unveiling the composition of relativistic jets of active galactic nuclei (AGNs) and gamma-ray bursts (GRBs), and the mechanisms of particle acceleration to ultrarelativistic energies within these jets, is among the prime outstanding issues in gamma-ray astronomy, as probed by the Fermi Gamma-Ray Space Telescope and ground-based Atmospheric Cherenkov Telescopes, such as H.E.S.S., MAGIC, and VERITAS, and the future Cherenkov Telescope Array. The physics of relativistic jets of AGNs is most directly probed by observations of blazars, whose jets are oriented at a small angle with respect to our line of sight. Their broadband nonthermal continuum emission consists of two broad emission components and is almost certainly produced in small, localized regions within the relativistic jet. It is commonly accepted that the radio through optical/UV (and in some cases X-ray) emission from blazars is synchrotron emission from relativistic particles. Leptonic models for the high-energy emission of blazars propose that the X-rays and gamma-rays from blazars are the result of Compton upscattering of lower-energy photons by the same relativistic electrons (see, e.g., Boettcher 2007 for a review of blazar emission models).

There are several lines of evidence that suggest the jets in blazars exhibit at least a two-component structure: a mildly relativistic, outer sheath with higher density, carries most of the kinetic energy of the jet, while a fast, highly relativistic inner spine of low comoving particle density carries most of the angular momentum. Direct observational evidence for radially structured spine-sheath jets comes from the limb-brightening of blazar and radio galaxy jets revealed in VLBI observations (Giroletti et al. 2004; Nagai et al. 2014). Prompted by such evidence, Ghisellini et al. (2005) proposed the radiative interaction between a fast, inner spine and a slower sheath in a blazar jet as a way to overcome problems with extreme bulk Lorentz factors required by spectral fits to several TeV BL Lac objects (Lyutikov \& Lister 2010). Hydrodynamic/MHD simulations of spine-sheath jets (Meliani \& Keppens 2007, 2009; Mizuno et al. 2007) indicate that the sheath, in combination with a poloidal magnetic field, aids in stabilizing the jet. Although Kelvin-Helmholtz-type instabilities (KHI, Chandrasekhar 1981) may develop at the spine-sheath interface and lead to turbulent mixing of the two phases, they may not disrupt the jet out to large distances from the central engine (Meliani \& Keppens 2007, 2009). The MHD turbulence developing at the spine-sheath interface of relativistic jets (Zhang et al. 2009) offers a promising avenue for relativistic particle acceleration in radio-loud AGNs and GRBs. However, the MHD approximation cannot directly address the creation of magnetic fields from unmagnetized shear flows or the acceleration of nonthermal particles.

The kinetic physics of relativistic shear flows has been successfully simulated using Particle-in-Cell (PIC, Birdsall \& Langdon 1991) simulations (Alves et al. 2012, 2014, 2015; Grismayer et al. 2013; Liang et al. 2013a, 2013b; Nishikawa et al. 2013, 2014, 2016). In our previous papers (Liang et al. 2013b, Liang et al. 2017), we have shown that ion-dominated relativistic shear flows lead to the creation of ordered dc electromagnetic (EM) fields near the shear boundary via the electron counter-current instability (ECCI), and the development of highly relativistic electron distributions peaking near the ion kinetic energy. However, those simulations assumed a high spine Lorentz factor $(\Gamma=451$ in the central engine frame, $p_{\mathrm{o}}=((\Gamma-1) / 2)^{1 / 2}=15$ in the center-of-momentum $(\mathrm{CM})$ frame). Hence those results are more relevant to GRBs (Liang et al. 2013b, 2017) than to AGNs.

In this paper, we present new PIC simulation results for a more moderate bulk Lorentz factor $\left(p_{\mathrm{o}}=5, \Gamma=51\right)$, relevant to radio-loud AGNs, in particular, blazars, in which bulk Lorentz factors $\Gamma \sim \mathrm{O}(10)$ are typically inferred from superluminal motion and radio brightness-temperature arguments (Jorstad et al. 2005; Hovatta et al. 2009). We will systematically compare the $p_{\mathrm{o}}=5$ shear boundary layer with the $p_{\mathrm{o}}=15$ shear boundary layer. To simplify the comparison, we first focus on pure electron-ion (e-ion) plasmas. Generalization to mixtures of e-ion and electron-positron (e+e-ion) plasmas does not alter our major conclusions, and will be briefly mentioned at the end of Section 2.

\section{Comparison of $p_{0}=5$ and $p_{0}=15$ Shear Boundaries}

As in our previous shear flow PIC simulations (Liang et al. 2013a, 2013b, 2016), we use the 2.5D (2D space, 


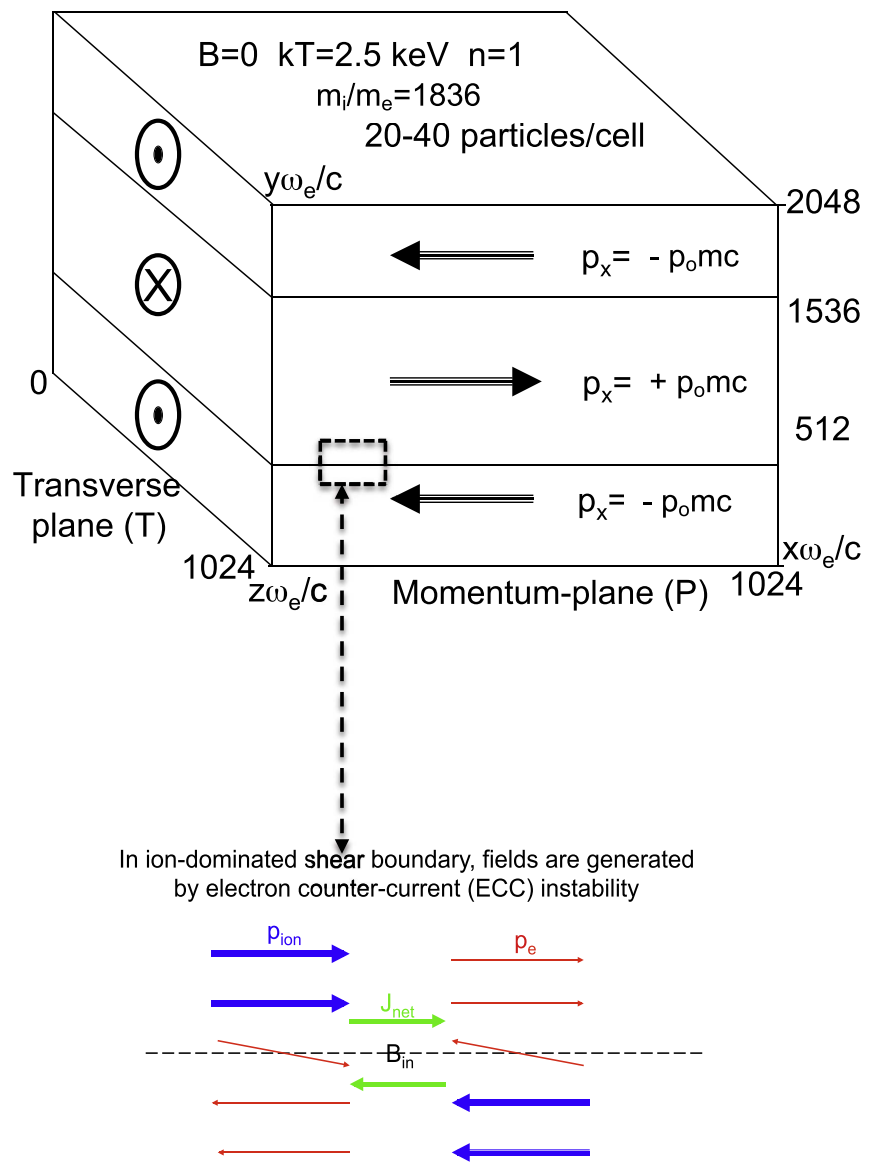

Figure 1. Setup of the (initially unmagnetized) shear flow PIC simulations of the e-ion plasma. This paper focuses on the longitudinal P-mode evolution in the $x-y$ plane only, since the transverse T-mode saturates at a very low level compared to the P-mode. In the present case the plasma consists of rightmoving plasma in the central $50 \%$ of the $y$-grid, referred to as the "spine," sandwiched between left-moving plasmas at the top $25 \%$ and bottom $25 \%$ of the $y$-grid, referred to as the "sheath." The simulation box has periodic boundary conditions on all sides. Inset: sketch illustrating d.c. magnetic field creation by the ECCI. Throughout this paper and in all figures, spatial scales are in units of the electron skin depth $c / \omega_{\mathrm{e}}$.

3-momenta) code Zohar-II (Birdsall \& Langdon 1991; Langdon \& Lasinski 1976) as the primary simulation tool. Though our Zohar-II simulation box is limited to $1024 \times 2048$ cells, this code has high numerical fidelity, and the numerical Cerenkov instability (NCI, Godfrey 1974, 1975) is strongly suppressed (Godfrey \& Langdon 1976). Hence it is well suited for simulations with relativistic particle drifts. In all iondominated shear flows, the T-mode (Liang et al. 2013a) in the $y-z$ plane transverse to the shear flow (Figure 1) saturates at very low amplitude compared to the P-mode (Liang et al. 2013a) in the $x-y$ plane parallel to the shear flow, and has negligible effects on the shear boundary structure (Liang et al. 2013b, confirmed by both 2.5D runs in the $y-z$ plane and $3 \mathrm{D}$ runs). Hence we focus on the 2D P-mode (Figure 1) results in the $x-y$ plane in this paper. All simulations are performed in, and all quantities below refer to, the $\mathrm{CM}$ frame. Periodic boundary conditions and initial temperature $k T=2.5 \mathrm{keV}$ for both electrons and ions $\left(m_{\mathrm{i}} / m_{\mathrm{e}}=1836\right)$. Throughout this paper and in all figures, distances are measured in units of electron skin depth $c / \omega_{\mathrm{e}}\left(\omega_{\mathrm{e}}=\right.$ electron plasma frequency) and times are measured in units of $1 / \omega_{\mathrm{e}}$. We normalize the initial density $n=1$ so that the cell size $=c / \omega_{\mathrm{e}}=1$ in dimensionless units. The plasmas are initially unmagnetized. Initially, right-moving plasma occupies the central $50 \%$ of the $y$-grid (hereafter called the "spine"), while initially left-moving plasma occupies the top $25 \%$ and bottom $25 \%$ of the $y$-grid (hereafter called the "sheath"; see Figure 1). To increase numerical stability, we used small time-step $\Delta t=0.1 / \omega_{\mathrm{e}}$. Overall energy conservation was better than $1 \%$.

We first compare the main features of $p_{\mathrm{o}}=5$ and $p_{\mathrm{o}}=15$ shear boundaries. Figure 2 shows the energy flows between ions, electrons, and EM fields for the two runs. We see that in both cases, the electron and ion energies reach full equipartition after $t \omega_{\mathrm{e}} \sim 9000$, and EM field energy saturates at $\sim 12 \%$ of total energy, showing that the e-ion energy equipartition and EM field energy saturation are insensitive to $p_{\mathrm{o}}$. Figure 3 compares the spatial profiles of $\boldsymbol{B}_{z}, \boldsymbol{E}_{y}, \boldsymbol{E}_{x}, \boldsymbol{J}_{x}$, and net charge $\rho=\left(n_{+}-n_{-}\right)$at $t \omega_{\mathrm{e}}=1000,3000$ and 12,000 , respectively, for the two runs. While the overall patterns are qualitatively similar, the shear boundary layers of the $p_{\mathrm{o}}=5$ case are thinner than those of the $p_{\mathrm{o}}=15$ case and the maximum values of the EM fields $\left(\boldsymbol{B}_{z}, \boldsymbol{E}_{y}\right)$ are much lower for $p_{\mathrm{o}}=5$ than $p_{\mathrm{o}}=15$ (Figure 3(a), (b)). Detailed analyses suggest that $\max (|\boldsymbol{B}|,|\boldsymbol{E}|)$ scale roughly as $p_{\mathrm{o}}^{3 / 2}$, and the boundary layer thickness scales roughly as $p_{0}$. Figure 4 compares the $x$ averaged density profiles of ions, electrons, and net charge as functions of $y$ for the two runs. This shows that the ion vacuum gap created by magnetic expulsion from the shear interface is present in both runs, but the ion vacuum gap is wider for $p_{\mathrm{o}}=15$ than for $p_{\mathrm{o}}=5$ due to stronger magnetic fields, with the gap width roughly proportional to $p_{\mathrm{o}}$. This robust ion vacuum gap is a unique feature of relativistic ion-dominated shear flows, which sustains the separation of the opposing flows and the long-term stability of the laminar boundary layer structure, preventing turbulent mixing of opposing ions. Electrons are evacuated less than the ions due to their mobility, leading to charge separation and the formation of a triple layer (double capacitor) at the shear boundary and associated $\boldsymbol{E}_{y}$ fields (see Figure 3(b)). Inductive $\boldsymbol{E}_{x}$ fields are generated parallel to the flow, adjacent to the boundary layer by $\partial \boldsymbol{B}_{z} / \partial \mathrm{t}$ (Figure 3(c)), which accelerates the electrons and decelerates the ions.

Figure 5 compares the electron and ion energy distributions for the two runs at late times. Because the bulk of particle acceleration/deceleration is done by the $\boldsymbol{E}_{x}$ fields, the artificial $y$-periodic boundary condition turns out to have little effect on the late-time electron and ion distributions, as we had previously demonstrated using much larger $y$-grids (Liang et al. 2013b, 2017). In both runs, the electron spectrum exhibits a narrow peak near the (decelerated) ion drift kinetic energy. In the $p_{\mathrm{o}}=5$ case, the electron spectrum peaks at $\gamma_{\mathrm{e}} \sim 3000$, consistent with the ion energy peak at $\sim 2.5 m_{\mathrm{i}} c^{2}$ (hence ion kinetic energy $\sim 1.5 m_{\mathrm{i}} c^{2}$ ). Similarly, for the $p_{\mathrm{o}}=15$ case, the electron spectrum peaks at $\gamma_{\mathrm{e}} \sim 14,000$, consistent with the ion energy peak at $\sim 7 m_{\mathrm{i}} c^{2}$ (Liang et al. 2013b). This confirms the scaling of the electron peak energy $\gamma_{\mathrm{e}}$ with $p_{\mathrm{o}}$. As we discuss below in Section 3, in the context of synchrotron models, the electron peak energy $\gamma_{\mathrm{e}}$ can be related to the synchrotron critical frequency (Rybicki \& Lightman 1979; 
(a) $\mathrm{p}_{\mathrm{o}}=5$

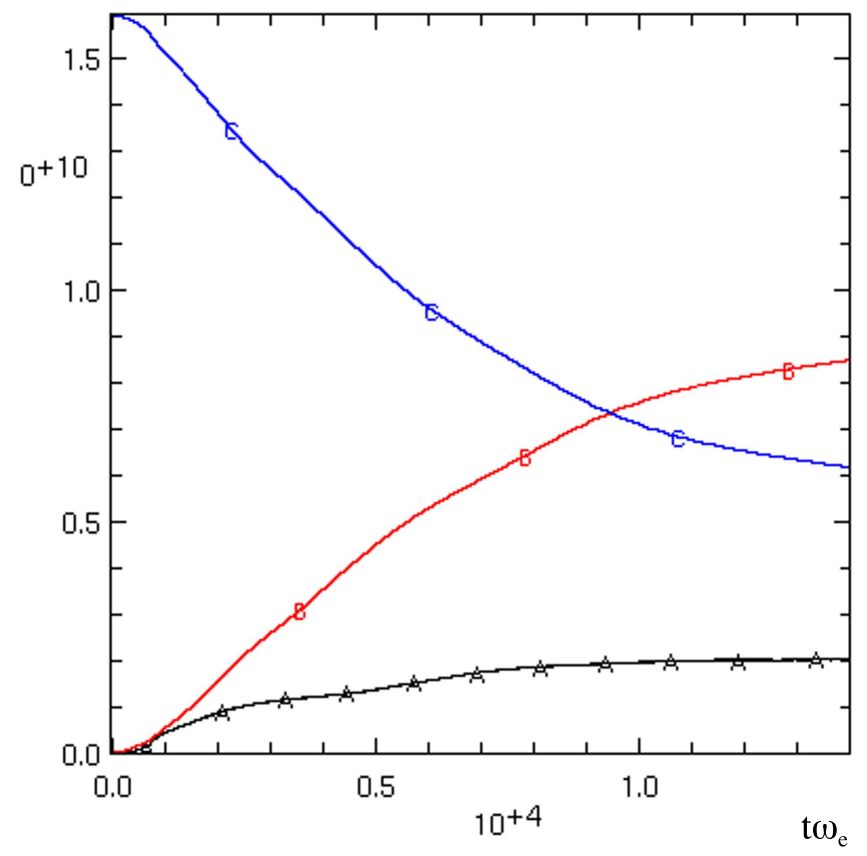

(b) $\mathrm{p}_{\mathrm{o}}=15$

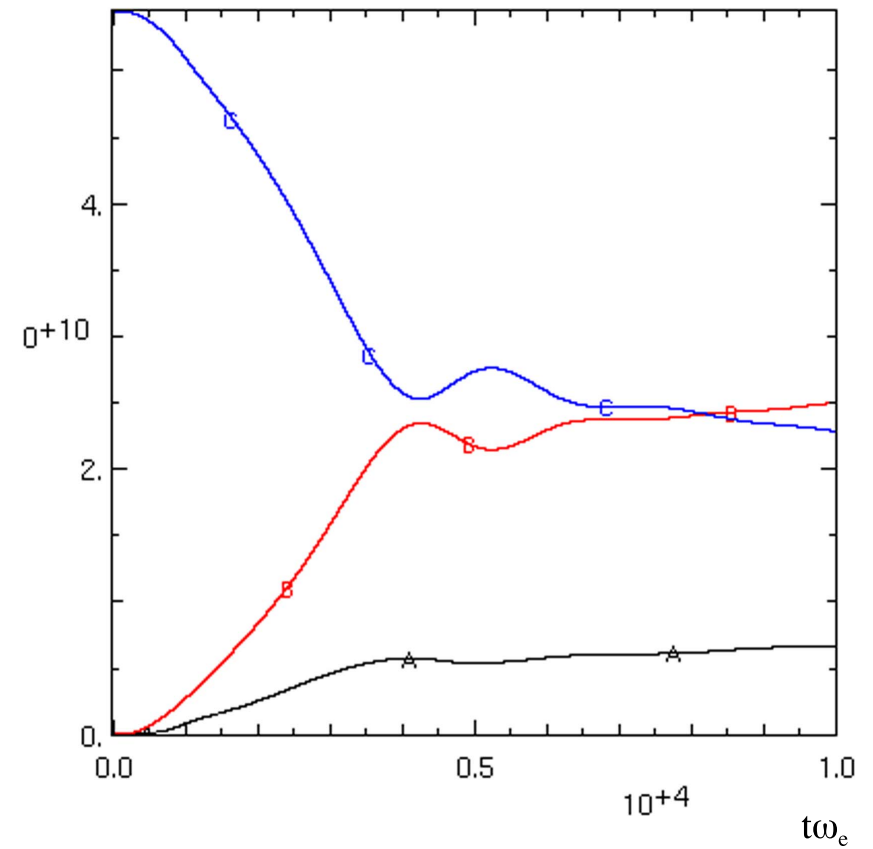

Figure 2. (a) Evolution of energy components of the $2 \mathrm{D}$ e-ion shear flow with $p_{\mathrm{o}}=5$ : EM field energy (A, black), electron energy (B, red), and ion energy (C, blue). At late times, the EM field energy saturates at $\sim 12 \%$ of total energy; (b) Evolution of energy components of the 2D shear flow with $p_{\mathrm{o}}=15$ : EM field energy (A, black), electron energy (B, red), ion energy ( $C$, blue). At late times the EM field energy also saturates at $\sim 12 \%$ of total energy. However, the electron energy approaches the ion energy faster in the $p_{\mathrm{o}}=15$ case than in the $p_{\mathrm{o}}=5$ case.

Sironi \& Spitkovsky 2009) via $\omega_{\mathrm{cr}} \sim \gamma_{\mathrm{e}}^{2} \omega_{\mathrm{B}}$, where $\omega_{\mathrm{B}}=\mathrm{e} B / m c$ is the electron gyrofrequency (=Lamor frequency). On the other hand, for Compton models, the inverse Compton peak is located at $\omega_{\mathrm{IC}} \sim \gamma_{\mathrm{e}}^{2} \omega_{\mathrm{o}}$, where $\omega_{\mathrm{o}}$ is the characteristic soft photon energy ( $\omega_{\mathrm{o}} \sim \omega_{\mathrm{cr}}$ for SSC models,
Boettcher 2007). Even though pure e-ion shear flows do not accelerate electrons much above the ion kinetic energy (Figure 5), when we add a moderate amount of $\mathrm{e}+\mathrm{e}-$ plasma into the e-ion plasma, a power-law tail eventually develops above $\gamma_{\mathrm{e}}$, due to the presence of nonlinear EM waves created outside the dc slab fields of Figure 3 (Liang et al. 2013b), which scatter the leptons stochastically to form the power-law tail. We observe that power-law tails develop in both the $p_{\mathrm{o}}=5$ and $p_{\mathrm{o}}=15$ hybrid $\mathrm{e}+\mathrm{e}-$ ion cases, but preliminary results suggest that the power-law slope may vary with both $p_{\mathrm{o}}$ and $\mathrm{e}+/$ ion ratio. Details remain to be investigated systematically.

\section{Applications to Blazars and GRBs}

Assuming that blazar and GRB jets indeed have a spinesheath structure, our shear boundary PIC simulation results above should be applicable to the local emission properties of the spine-sheath interface. To better visualize the differences in particle momentum distribution and radiation characteristics between the $p_{\mathrm{o}}=5$ and $p_{\mathrm{o}}=15$ cases, it is better to Lorentz boost the particle momenta from the CM frame of Section 2 back to the "laboratory" frame (LF) in which the sheath is initially at rest, and the spine moves with the bulk Lorentz factor $\Gamma=2 p_{o}^{2}+1$. Figures 6 and 7 compare various phase plots for the two runs, after Lorentz boosting (in the $-\boldsymbol{x}$ direction) from the CM frame back to the LF. We see that for $p_{\mathrm{o}}=5$ (Figures 6(a), 7(a)), spine electrons are accelerated to peak at $\gamma_{\mathrm{Lab}} \sim 30,000$ or $15 \mathrm{GeV}$, whereas for $p_{\mathrm{o}}=15$ (Figures 6(b), 7(b)), spine electrons are accelerated to peak at $\gamma_{\text {Lab }} \sim 4.4 \times 10^{5}$ or $220 \mathrm{GeV}$. The highest-energy spine electron momenta achieve more extreme anisotropy $\left(\boldsymbol{p}_{x \mathrm{Lab}} \ggg \boldsymbol{p}_{y}\right)$ for $p_{\mathrm{o}}=15$ than for $p_{\mathrm{o}}=5$, while the beam angle $\left|\boldsymbol{p}_{y} / \boldsymbol{p}_{x \text { Lab }}\right|$ decreases exponentially with increasing energy for both $p_{\mathrm{o}}=5$ and $p_{\mathrm{o}}=15$ (Figure 8). In fact, on average, both beam angles are much narrower than simple Doppler boosting of an isotropic distribution in the spine rest frame to the LF $(1 / \Gamma$, red dashed line). Observationally, we therefore expect GRB jets to emit much harder radiation with narrower beaming and more rapid time variability than blazar jets, and the photon energy should be correlated with time variability and anti-correlated with beam angle. Such observational predictions of shear boundary emission should be testable.

In summary, our PIC simulation results show that efficient lepton acceleration up to $\gamma_{\mathrm{e}} \sim \Gamma m_{\mathrm{i}} / m_{\mathrm{e}}$ occurs in relativistic shear boundary layers, and proceeds in a strongly anisotropic manner. The highest-energy leptons are beamed into an angle much narrower than $1 / \Gamma$ in the laboratory frame. In the process of Compton scattering by relativistic leptons, the scattered, high-energy photon emerges in the direction of the scattering lepton. Hence jets viewed in the direction tangential to the shear boundary will exhibit very hard radiation spectra, far beyond the usual spectral hardening effect due to bulk Doppler boosting of a comoving isotropic particle distribution (which is just a shift of the peak frequency by factor $\Gamma$ ). On the other hand, jets viewed at substantial off-axis angles (assuming that the shear layer is largely parallel to the global jet axis) will exhibit softer spectra. These beaming effects should become more acute for GRBs (Meszaros 2002; Piran 2004; Preece et al. 1998) than blazars, and more extreme for the Compton peak than the synchrotron peak. The narrow beaming may also explain the minute-scale rapid time 

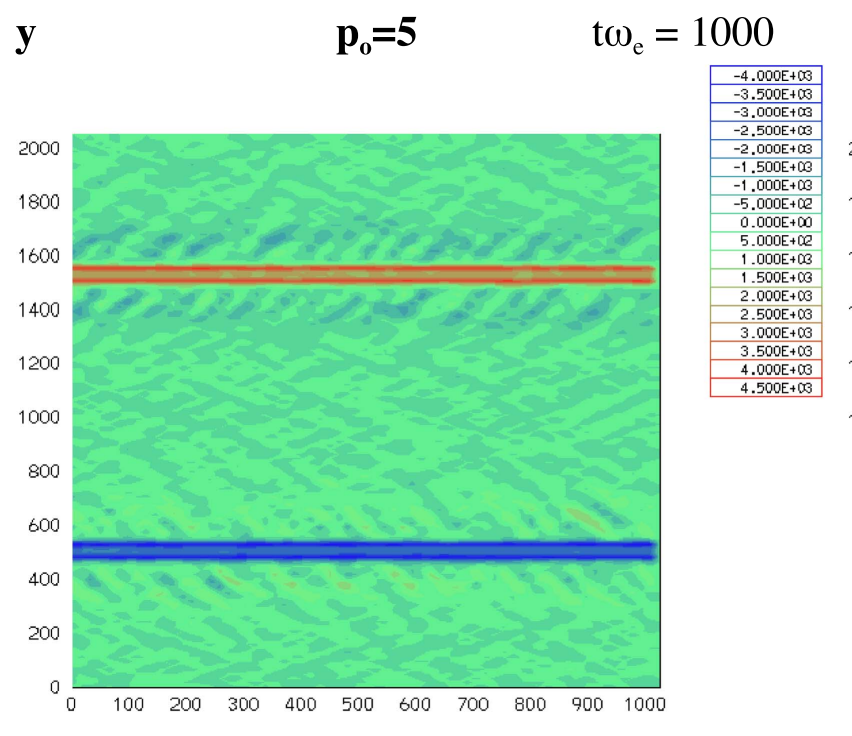
$\mathbf{y}$
$p_{0}=15$

$\mathbf{y}$

$\mathrm{t} \omega_{\mathrm{e}}=3000$
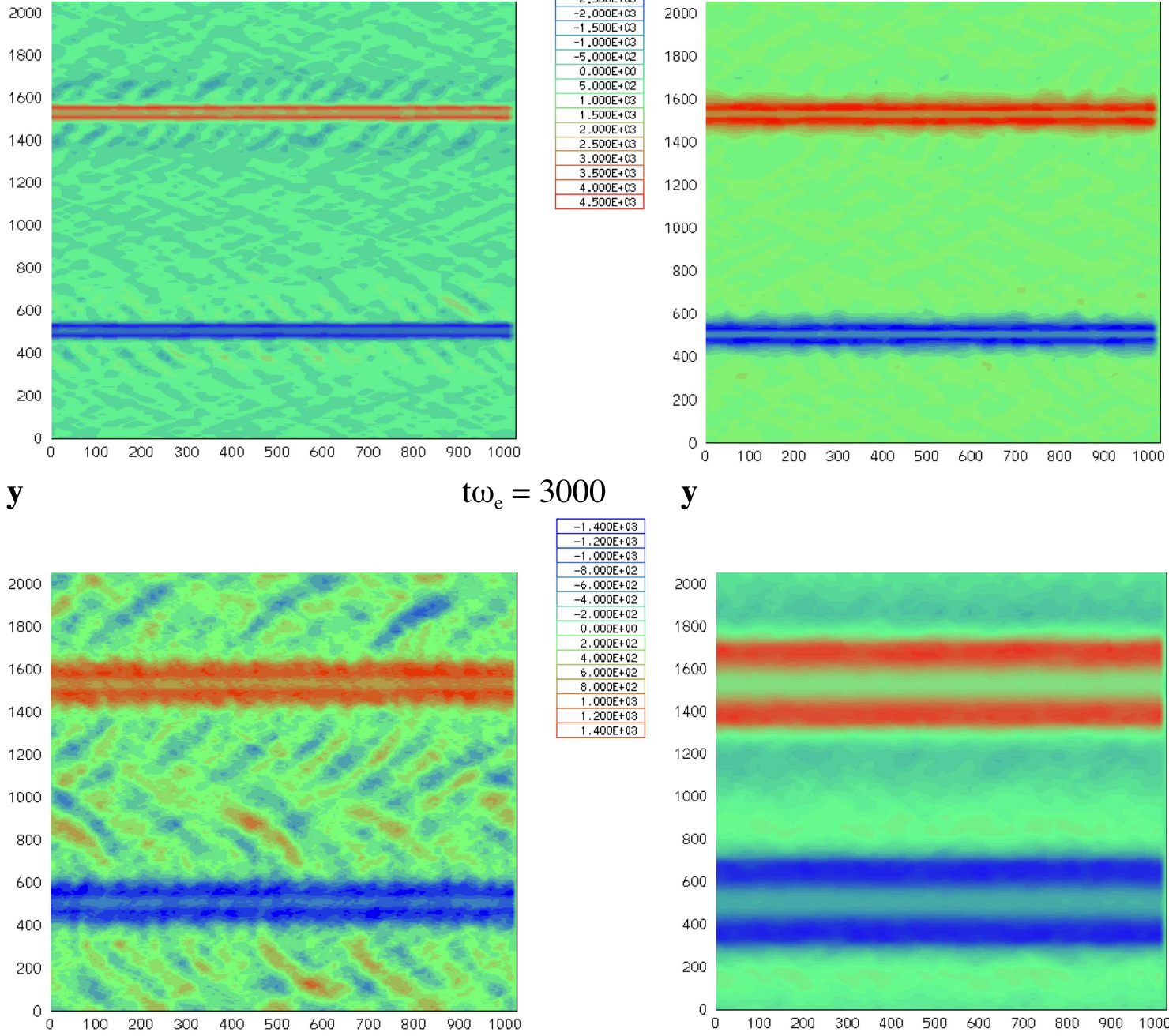

1600

1400

1200

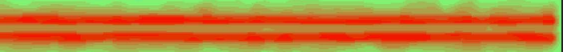

200

1000

800

600

400
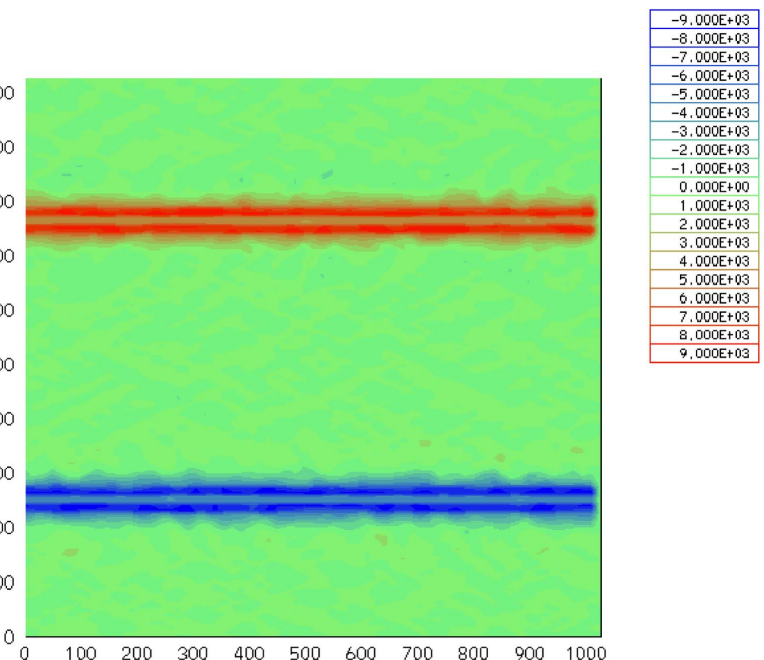

$\mathbf{y}$
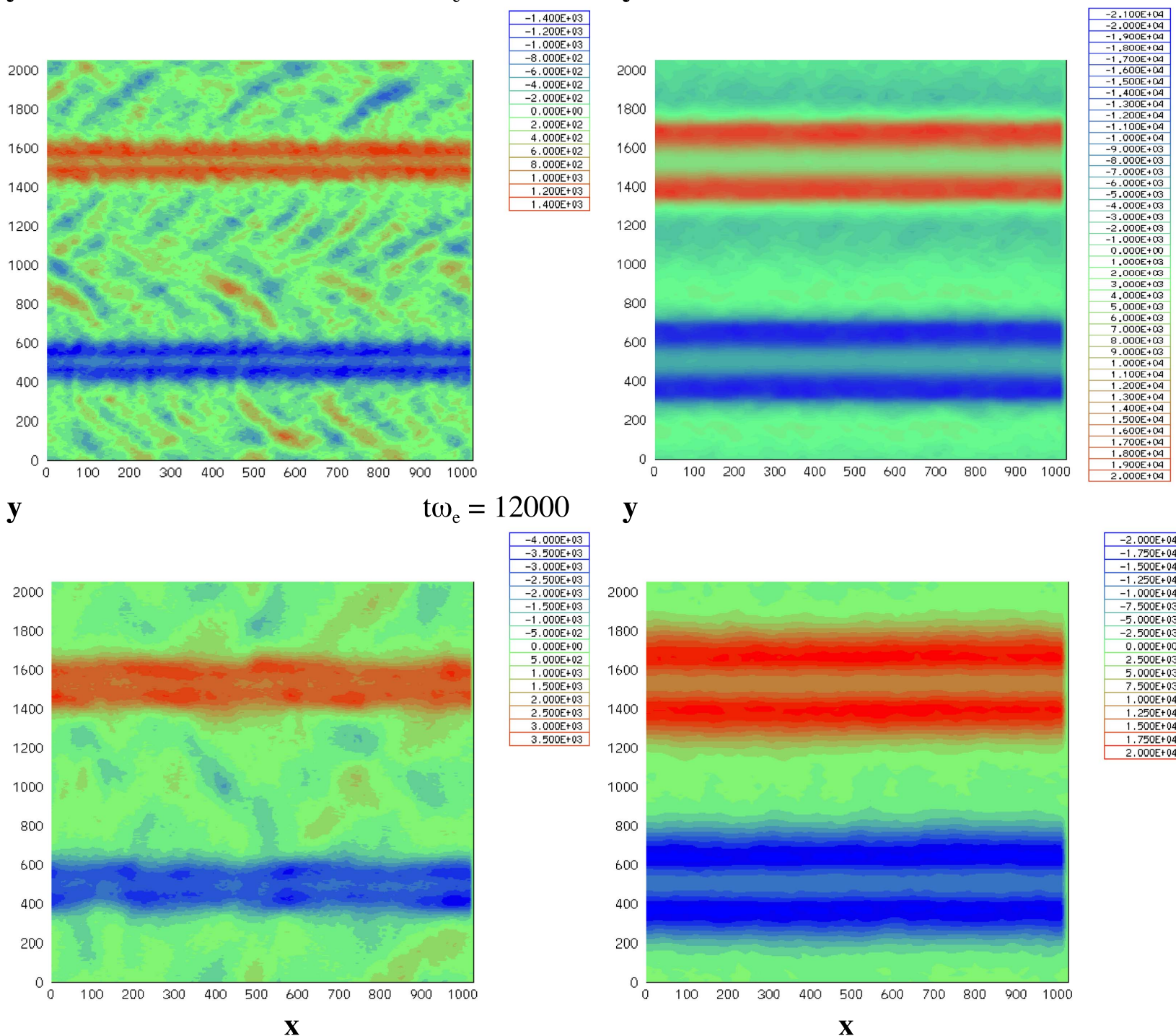

2000

$\mathbf{y}$
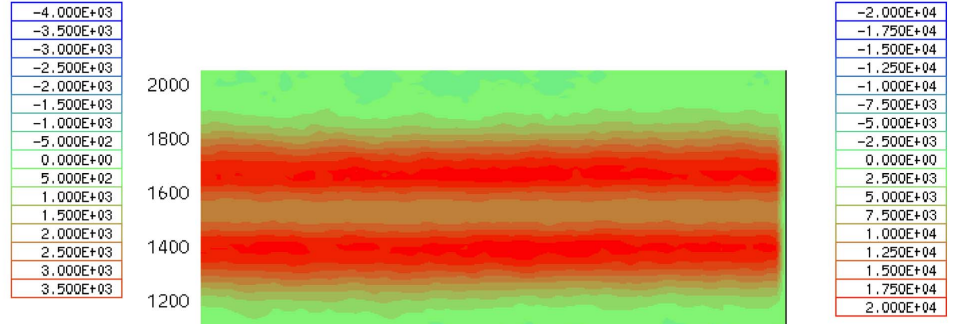

(a) $\mathbf{B}_{\mathbf{z}}$

Figure 3. Comparison of spatial profiles of (a) $\boldsymbol{B}_{z}$, (b) $\boldsymbol{E}_{y}$, (c) $\boldsymbol{E}_{x}$, (d) $\boldsymbol{j}_{x}$, (e) $\rho=$ net charge $=\left(n_{\mathrm{i}}-n_{\mathrm{e}}\right.$ ), between $p_{\mathrm{o}}=5$ (left columns) and $p_{\mathrm{o}}=15$ (right columns) cases at three different times: $t \omega_{\mathrm{e}}=1000$ (top), 3000 (middle), 12,000 (bottom). We note that the average shear boundary layer thickness of the $p_{\mathrm{o}}=5$ case is $\sim 1 / 3$ that of the $p_{\mathrm{o}}=15$ case, scaling roughly as $\sim p_{\mathrm{o}}$. The maximum $\boldsymbol{B}_{z}, \boldsymbol{E}_{y}$ fields at $t w_{e}=12000$ of the $p_{\mathrm{o}}=5$ case is lower than those of the $p_{\mathrm{o}}=15$ case, scaling roughly as $p_{o}^{3 / 2}$. 


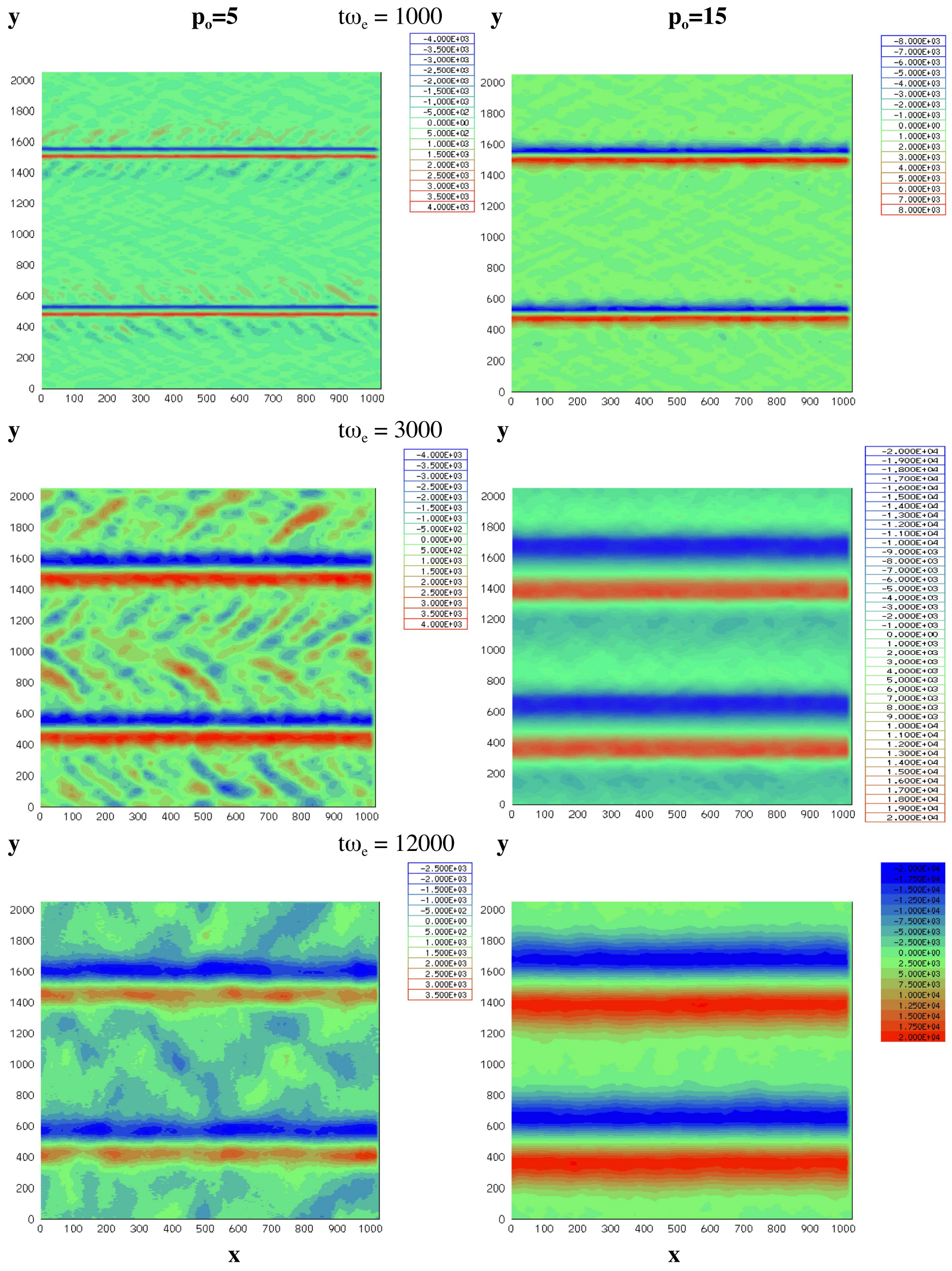

(b) $\mathbf{E}_{\mathbf{y}}$

Figure 3. (Continued.) 

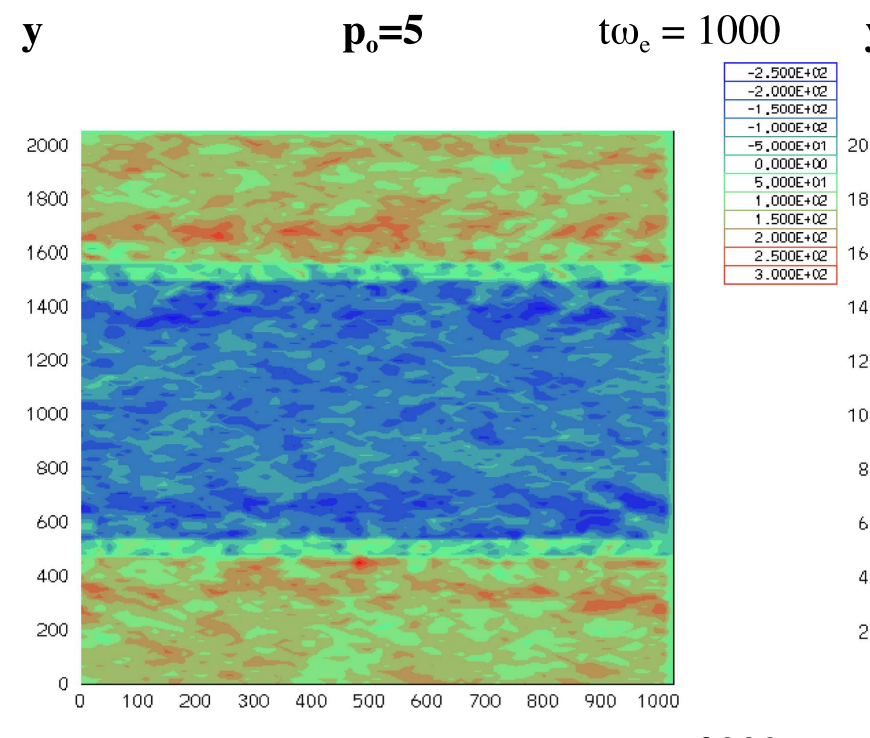
$\mathbf{y}$
$p_{0}=15$

$\mathbf{y}$

$\mathrm{t} \omega_{\mathrm{e}}=3000$
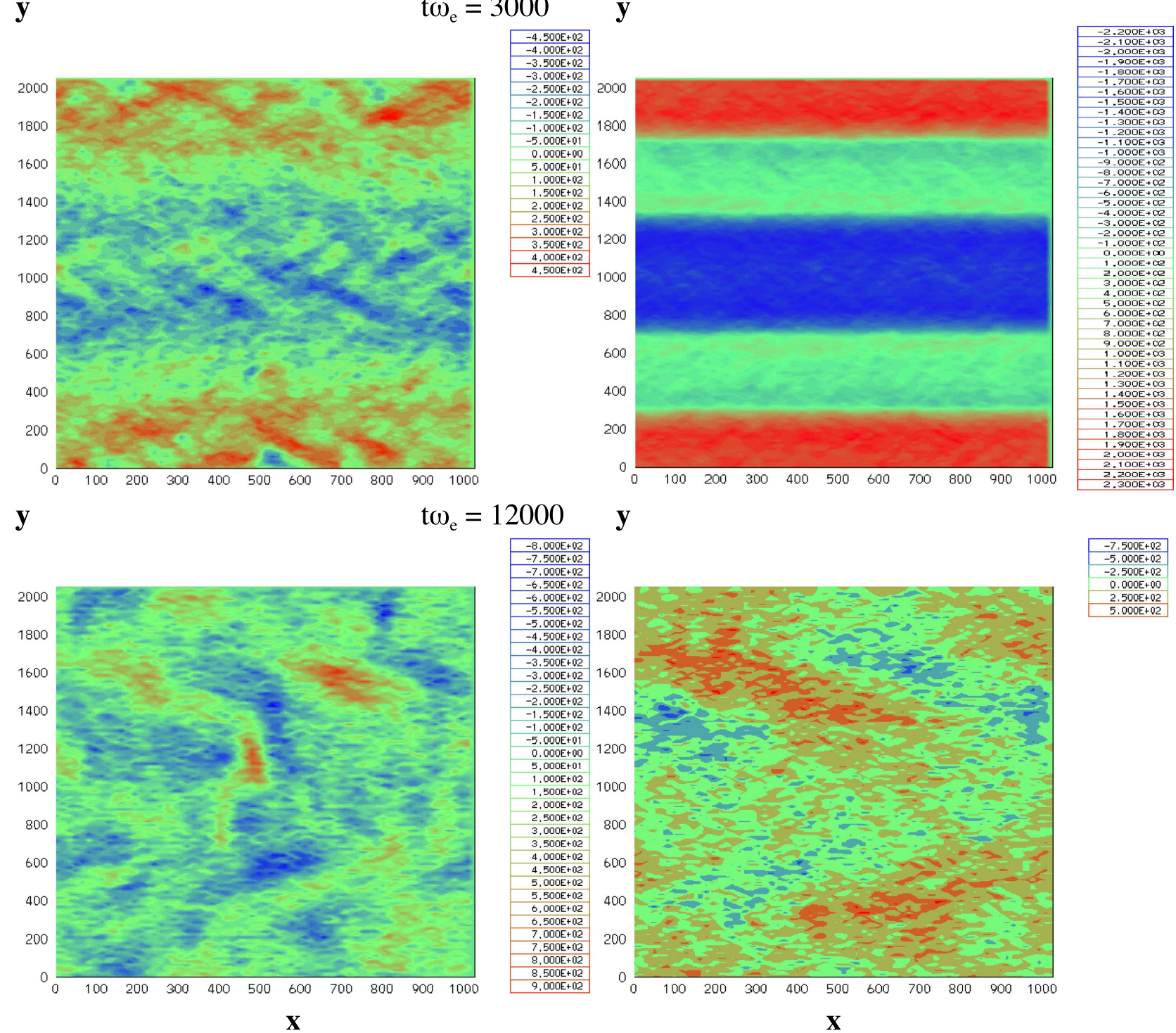

(c) $\mathbf{E}_{\mathbf{x}}$

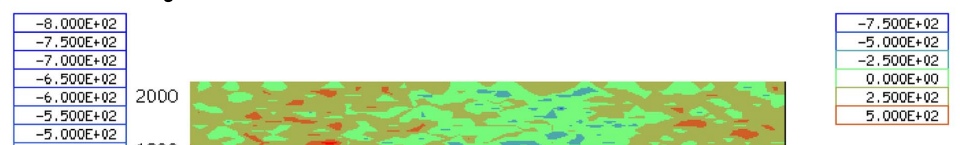

Figure 3. (Continued.) 


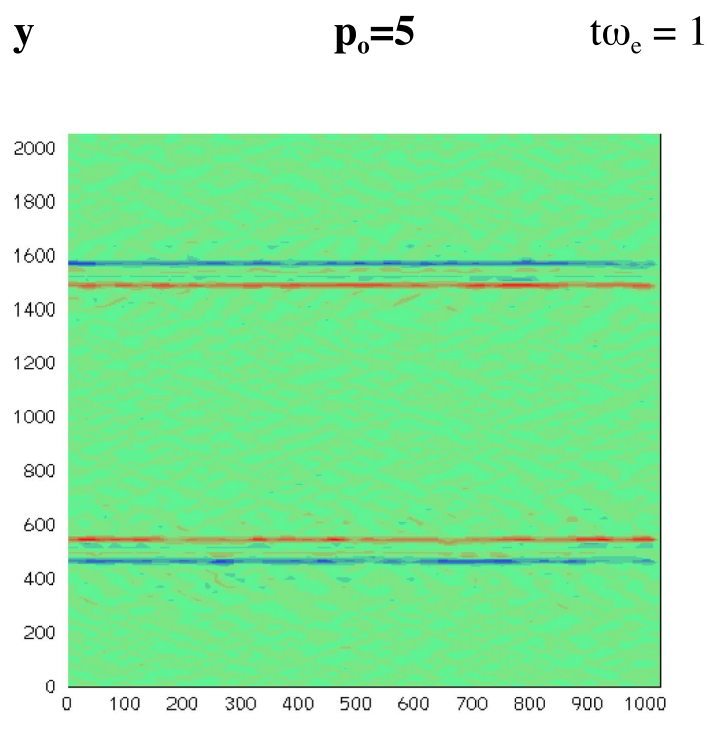

$\begin{array}{lll}\mathbf{y} & \mathbf{p}_{\mathbf{o}}=\mathbf{1 5}\end{array}$
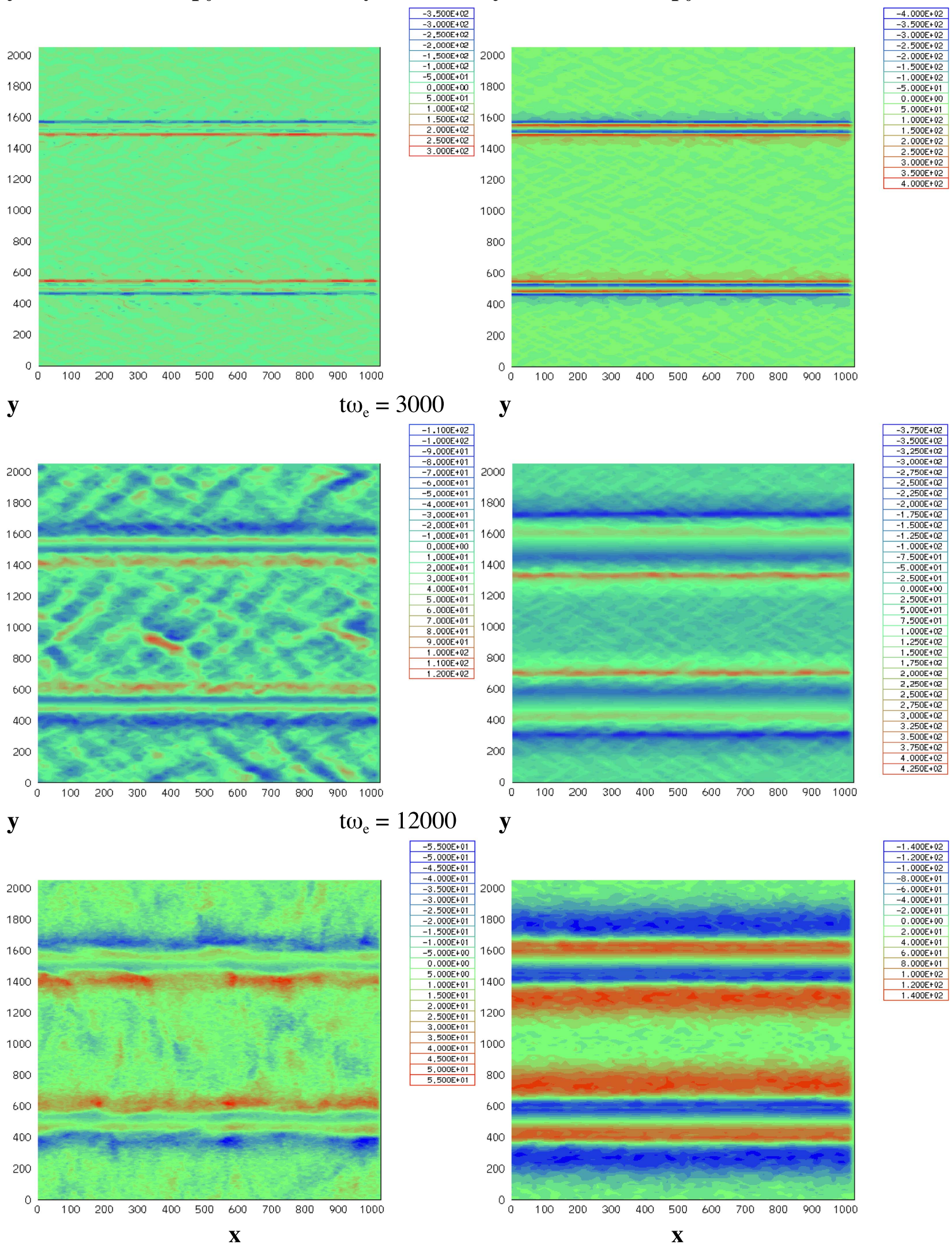

(d) $\mathbf{j}_{\mathbf{x}}$
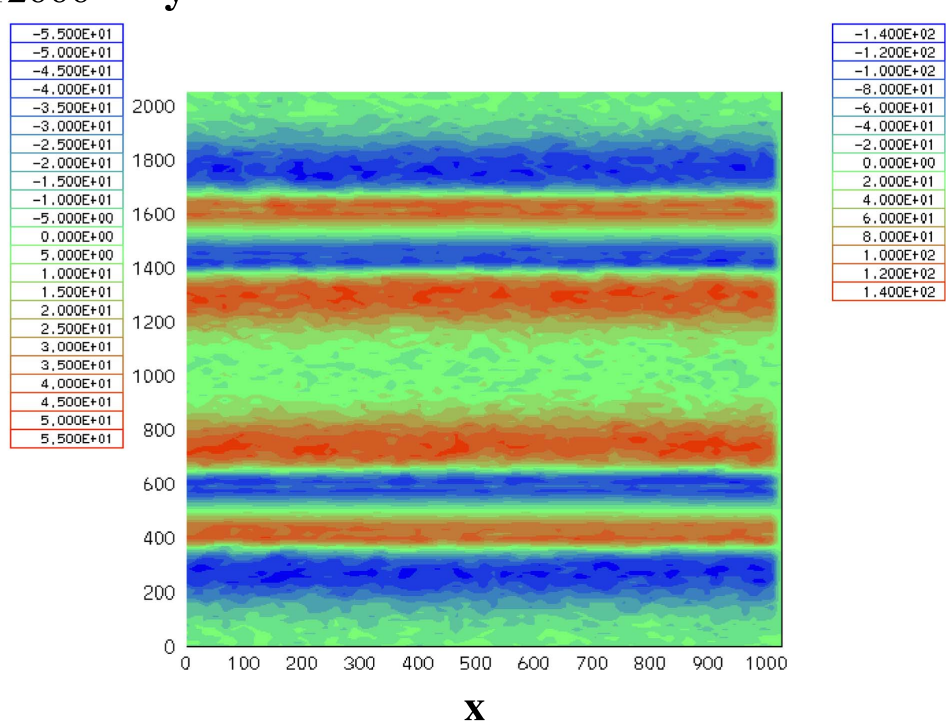

Figure 3. (Continued.) 


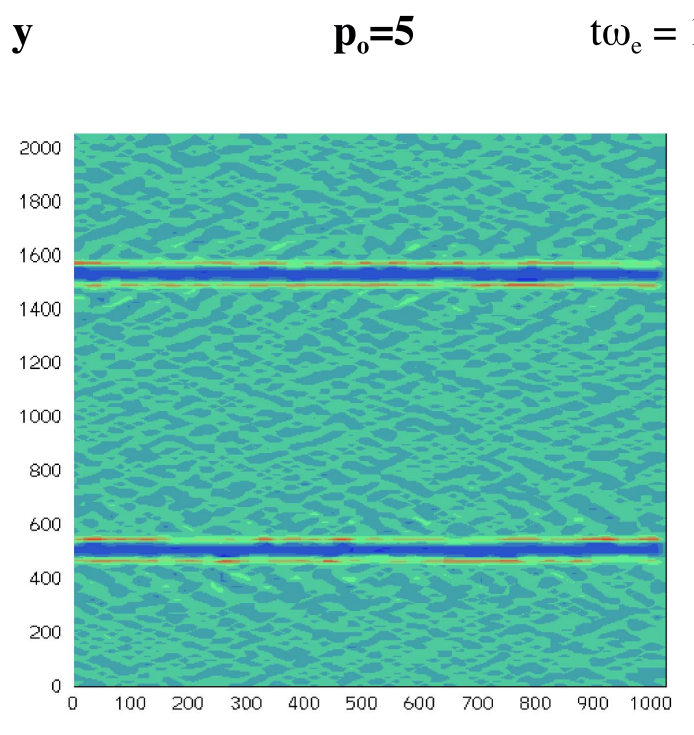

$1000 \quad \mathbf{p} \quad \mathbf{p}_{\mathbf{0}}=\mathbf{1 5}$
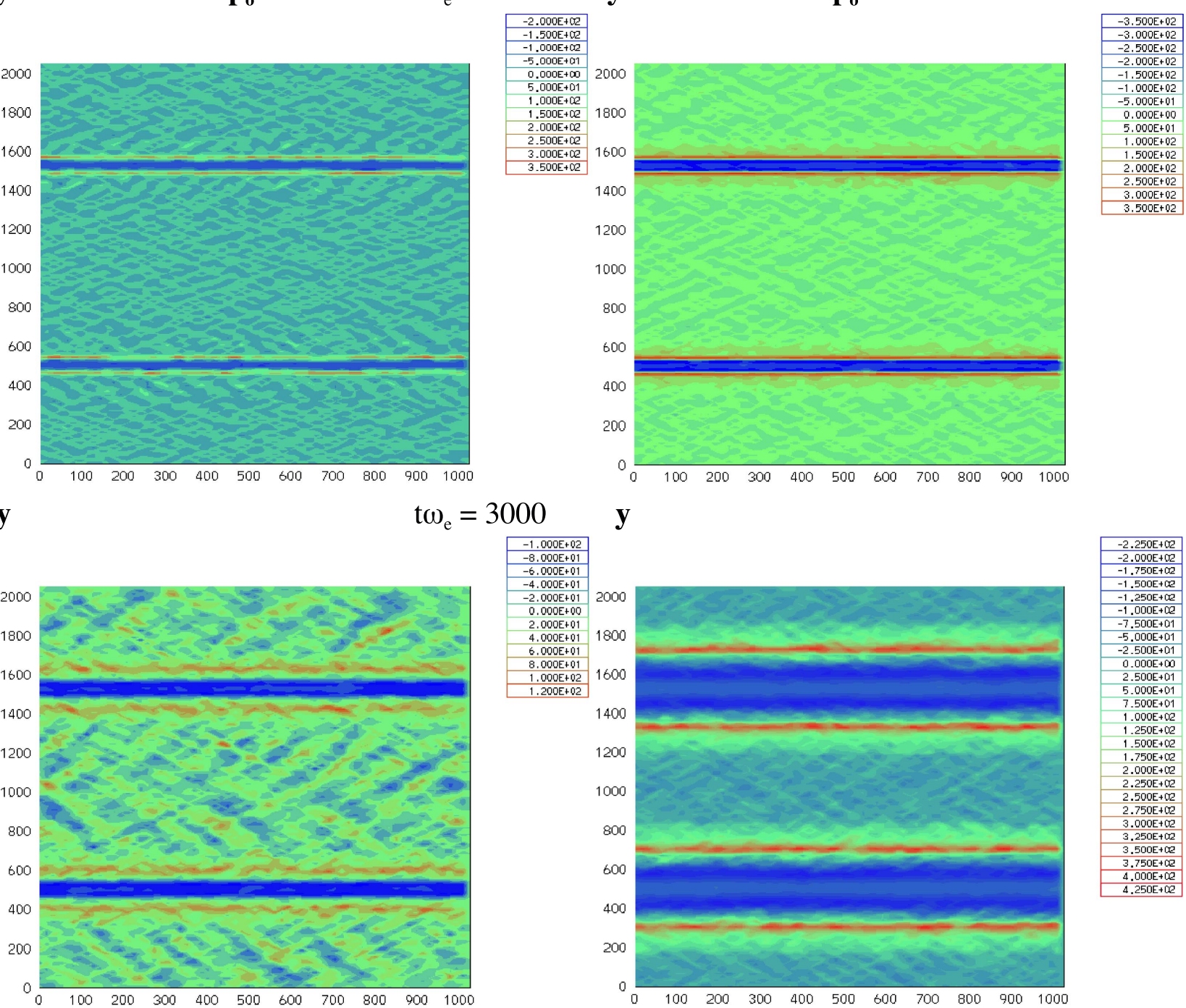

3000

$\mathbf{y}$

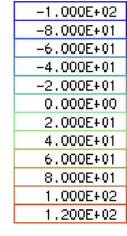

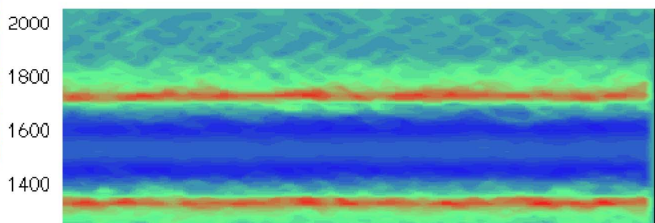

1400

1200

1000

800

600
400
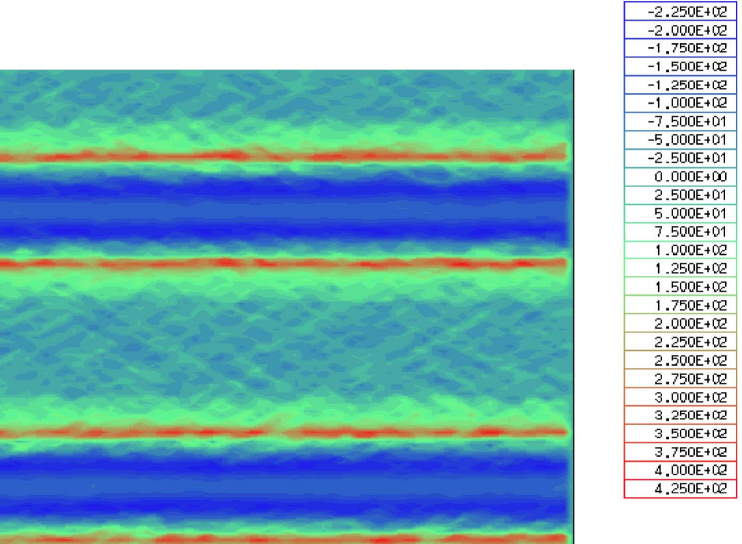

200

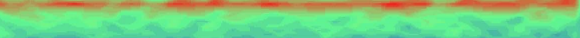

$2505+\infty$

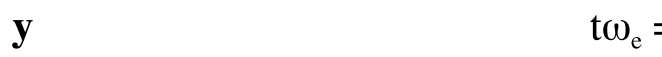

2000
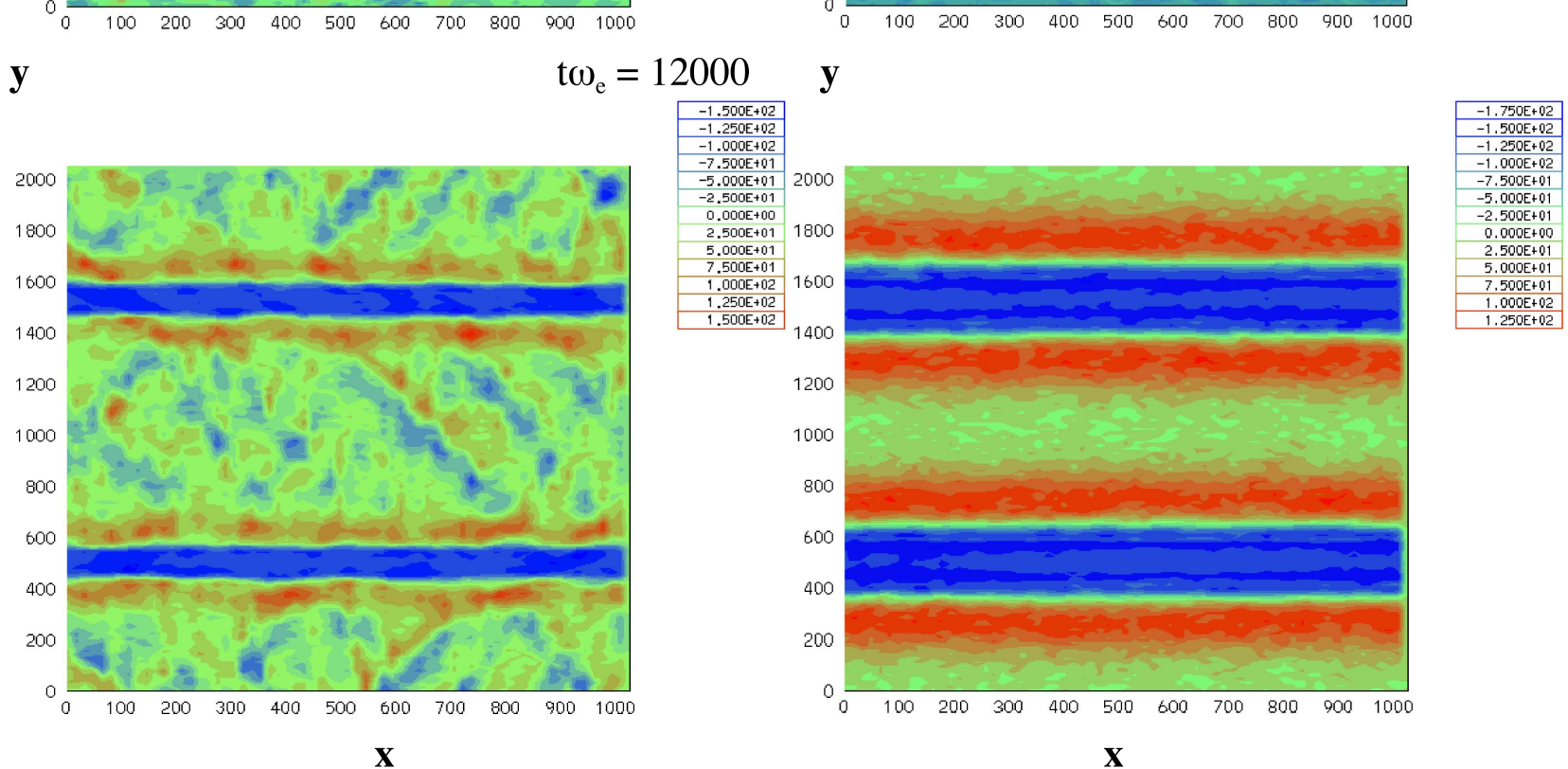

(e) $\rho$

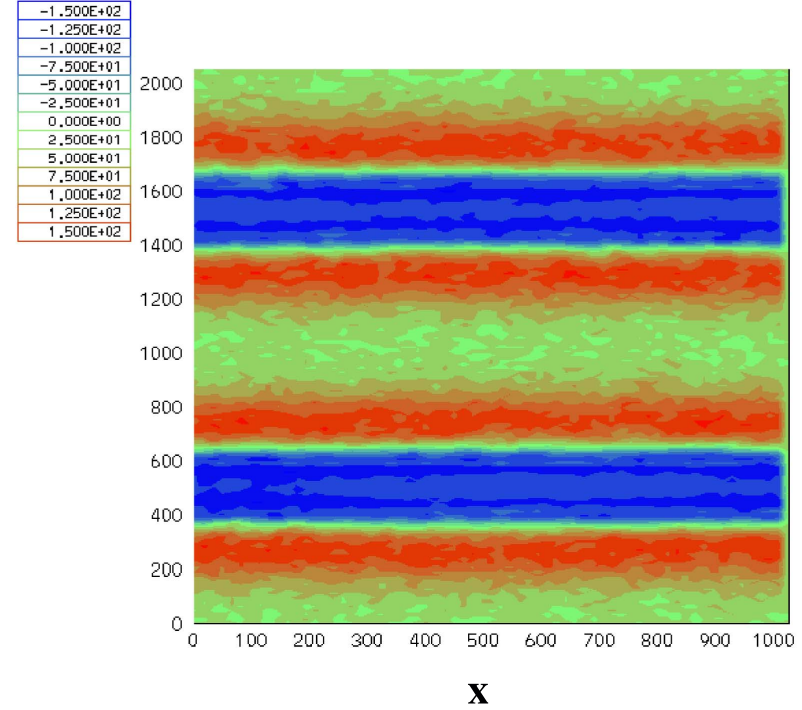

Figure 3. (Continued.) 


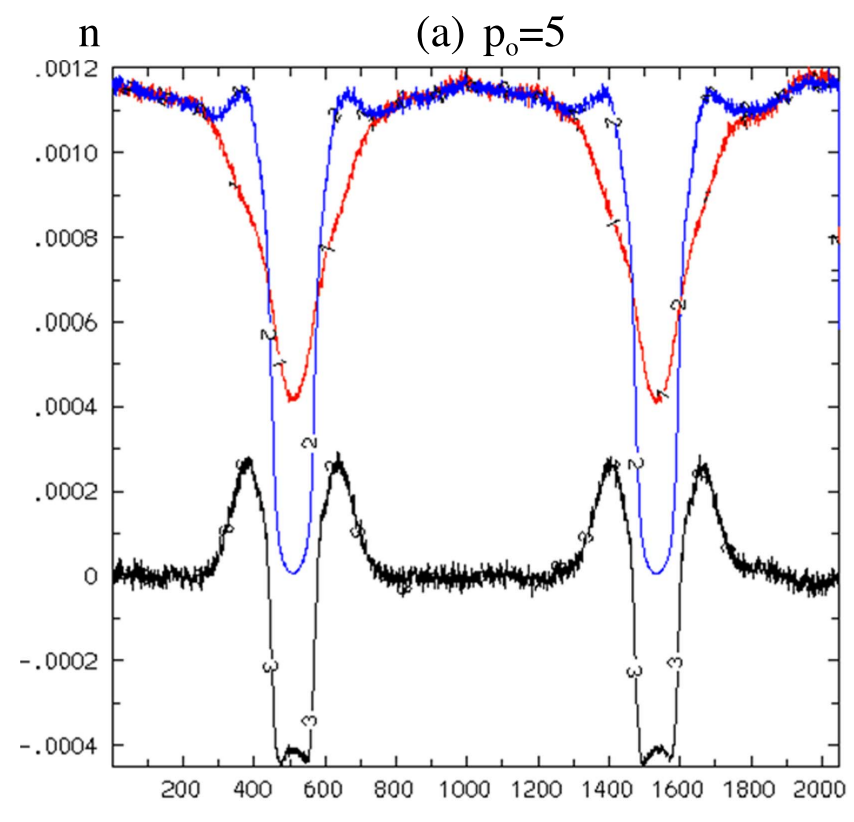

$\mathrm{y}$

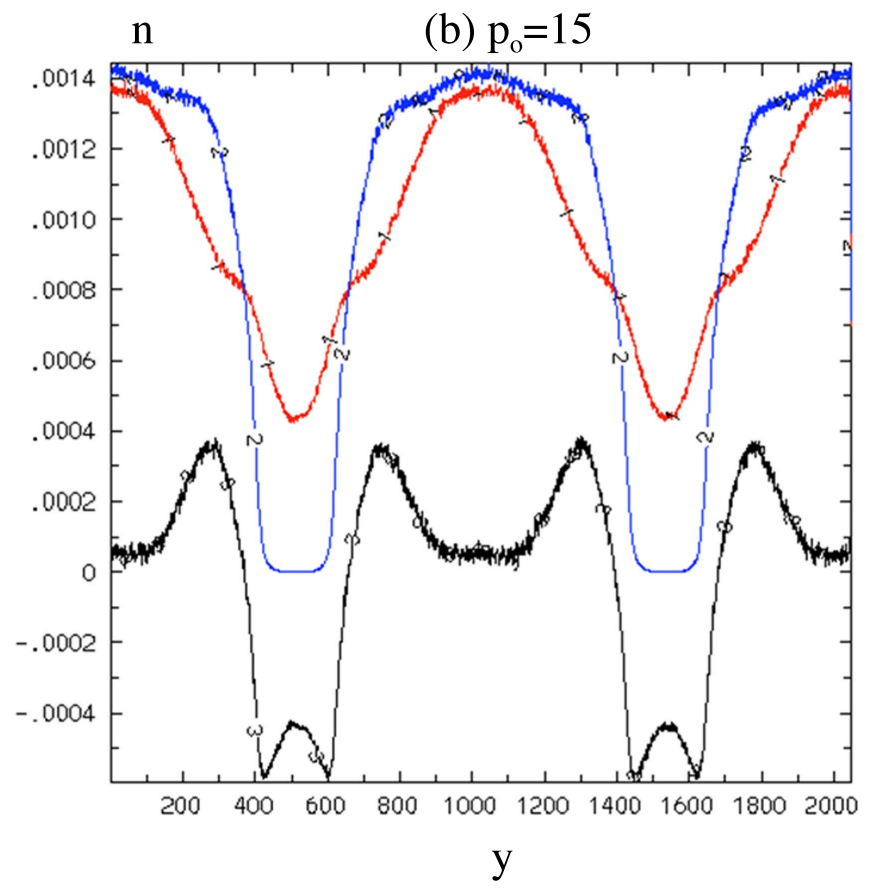

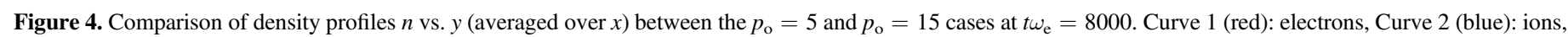

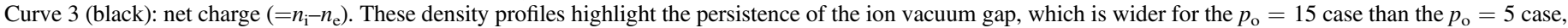

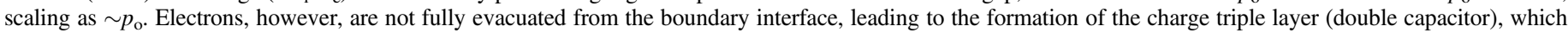
plays an important role in electron energization.

variability of some blazars (F. Tavecchio \& G. Ghisellini, private communication).

The results presented above indicate that relativistic shear layers in ion-dominated plasmas are capable of producing relativistic electron distributions in the $\mathrm{CM}$ frame with pronounced peaks at $\gamma_{\mathrm{e}} \sim$ few $\times 10^{3}$ for blazar Lorentz factors $\Gamma \sim 10$. In the presence of a magnetic field of $B=B_{\mathrm{G}}$ Gauss in the $\mathrm{CM}$ frame, this results in an observed synchrotron peak frequency of $\nu \sim 10^{14} B_{\mathrm{G}} \mathrm{Hz}$ in the LF, typically observed in low-synchrotron-peaked (LSP) blazars, i.e., flat-spectrum radio quasars and low-frequency-peaked BL Lac objects.

These same electrons will then also produce gamma-rays via Compton upscattering of the cospatially produced synchrotron photons (the synchrotron self-Compton (SSC) process) and possibly photons produced external to the jet (the external-Compton (EC) process), e.g., in the broad-line region or infrared-emitting dusty torus around the central accretion flow (Boettcher et al. 2013). Synchrotron photons can be up-scattered (SSC) in the Thomson regime, which is expected to be the case for blazars for any plausible magnetic-field value. This will then result in a peak photon energy of the SSC emission of $\sim$ few $\mathrm{MeV} B_{\mathrm{G}}$. Gamma-ray emission of LSP blazars is often dominated by SSC emission (Boettcher et al. 2013). External photons with stationaryframe energy $\mathrm{h} \nu_{\mathrm{o}} \sim \mathrm{eV}$ will be Compton up-scattered in the Klein-Nishina regime to yield maximum observed photon energies of $\sim 15 \mathrm{GeV}$ in LF. This is consistent with the gamma-ray peaks in LSP blazars typically being located at $100 \mathrm{MeV}$ to $\mathrm{GeV}$.

These estimates illustrate that for characteristic values of bulk Lorentz factor $\Gamma \sim 10$, the shear boundary energization scenario predicts synchrotron peaks in the IR-optical and EC gamma-ray peaks up to the $\mathrm{GeV}$ regime, as typically observed in LSP blazars (Abdo et al. 2010), along with SSC-dominated hard X-ray and soft gamma-ray emission, peaking around $\sim$ few $\mathrm{MeV}$. However, applying this scenario to high-frequencypeaked BL Lac objects (HBLs) with observed synchrotron peak frequencies of $\nu \sim 10^{17} \mathrm{~Hz}$ would require bulk Lorentz factors much higher than typical values of $\Gamma \sim 10$ inferred for blazars, in general, unless the magnetic field in the CM frame is $\gg$ Gauss. If the jet composition is dominated by ions in both the spine and the sheath as assumed in this paper, one expects a small population of AGNs viewed under very small viewing angles with $\theta_{\text {obs }} \ll 1 / \Gamma$, with very hard gamma-ray spectra, while a larger population of off-axis AGNs with $\theta_{\text {obs }}$ $>1 / \Gamma$ appear to have much softer spectra (see Figure 8). For the small population viewed at $\theta_{\mathrm{obs}} \ll 1 / \Gamma$, the radiation will be dominated by a narrow population of electrons with Lorentz factors $\gamma_{\text {eLab }} \sim$ few $\times 10^{4}$ (Figure 6(a), Figure 7(a)), thus producing very hard synchrotron and Compton spectra peaking at significantly higher energies than those given by the estimates above. The recently emerging class of extreme BL Lac objects (e.g., Bonnoli et al. 2015) may possibly represent the small population of extremely narrowly beamed, very-hard-spectrum blazars expected in the ion-dominated shear flow scenario.

This work was partially supported by NSF AST1313129 and Fermi Cycles 4 and 5 GI grants to Rice University, and NASA Fermi GI Grant no. NNX12AE31G to Ohio University. The work of M.B. is supported through the South African Research Chairs Initiative (SARChI) of the Department of Science and Technology and the National Research Foundation of South Africa under SARChI Chair grant no. 64789. We thank Drs. Fabrizio Tavecchio and Gabriele Ghisellini for useful discussions. Simulations with the ZoharII code were supported by the Lawrence Livermore National Laboratory. 


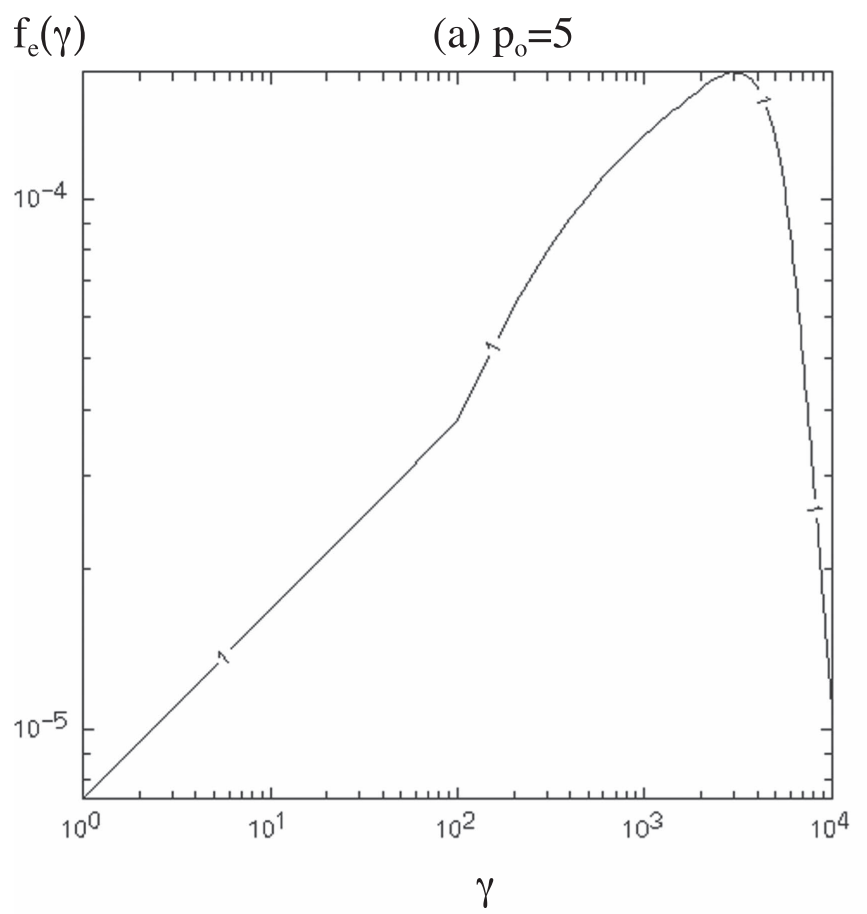
$\mathrm{f}_{\mathrm{e}}(\gamma)$
(b) $p_{0}=15$
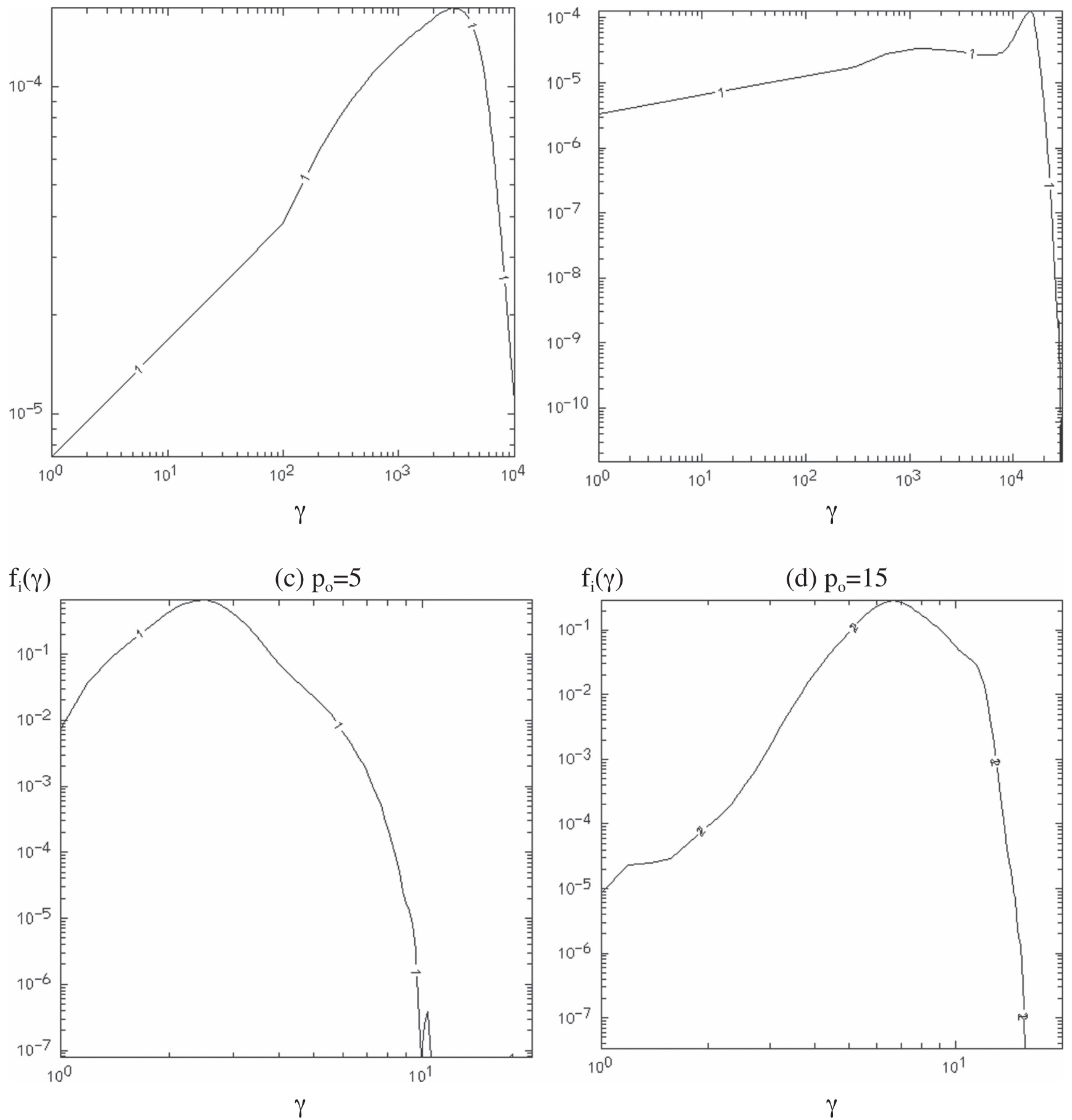

Figure 5. Comparison of electron distribution function $f_{\mathrm{e}}(\gamma)$ (particle no. per unit $\gamma$ ) vs. $\gamma$ in CM frame for (a) the $p_{\mathrm{o}}=5$ case with that of (b) the $p_{\mathrm{o}}=15$ case at $t \omega_{\mathrm{e}}=10,000$; (c), (d) Same as (a), (b) for ion distribution function $f_{\mathrm{i}}(\gamma)$ at $t \omega_{\mathrm{e}}=10,000$. We see that the electron energy peaks at $\gamma_{\mathrm{e}} \sim \gamma_{\mathrm{i}} m_{\mathrm{i}} / m_{\mathrm{e}}$ in both cases. 

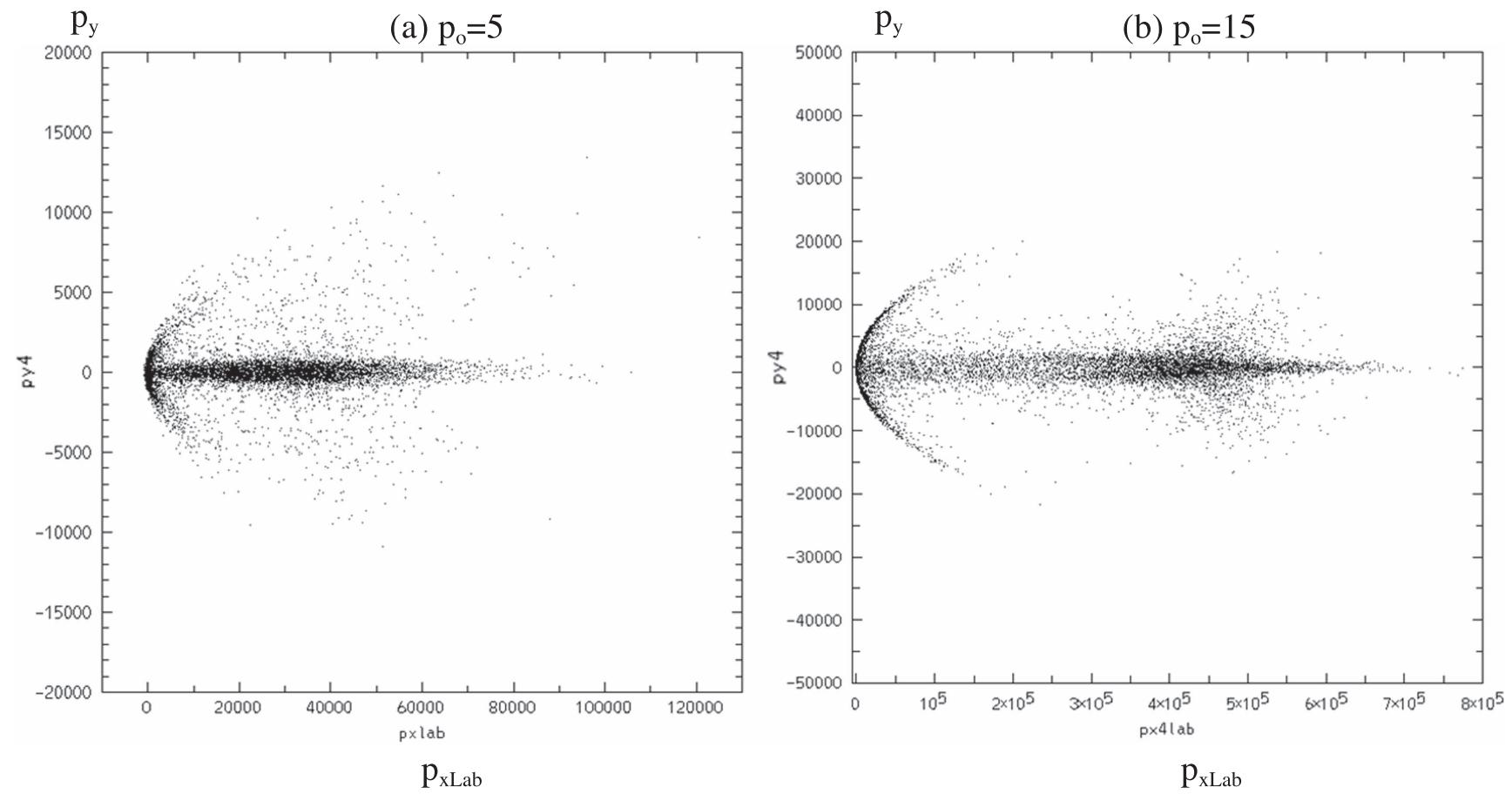

Figure 6. Phase plot $\left(\boldsymbol{p}_{y}\right.$ vs. $\left.\boldsymbol{p}_{x \mathrm{Lab}}\right)$ of spine electrons for the $p_{\mathrm{o}}=5$ case (a) compared to that of the $p_{\mathrm{o}}=15$ case (b) at $t \omega_{\mathrm{e}}=8000$, after Lorentz boosting $\boldsymbol{p}_{x}$ to the "laboratory frame" in which the sheath is initially at rest. By this time, some of the spine electrons have diffused into the sheath region and are decelerated, forming the low-energy bow-shaped population at left. The arrow-shaped high-energy population corresponds to electrons remaining in the spine. Note that electrons are more concentrated at the highest energy for the $p_{\mathrm{o}}=15$ case.
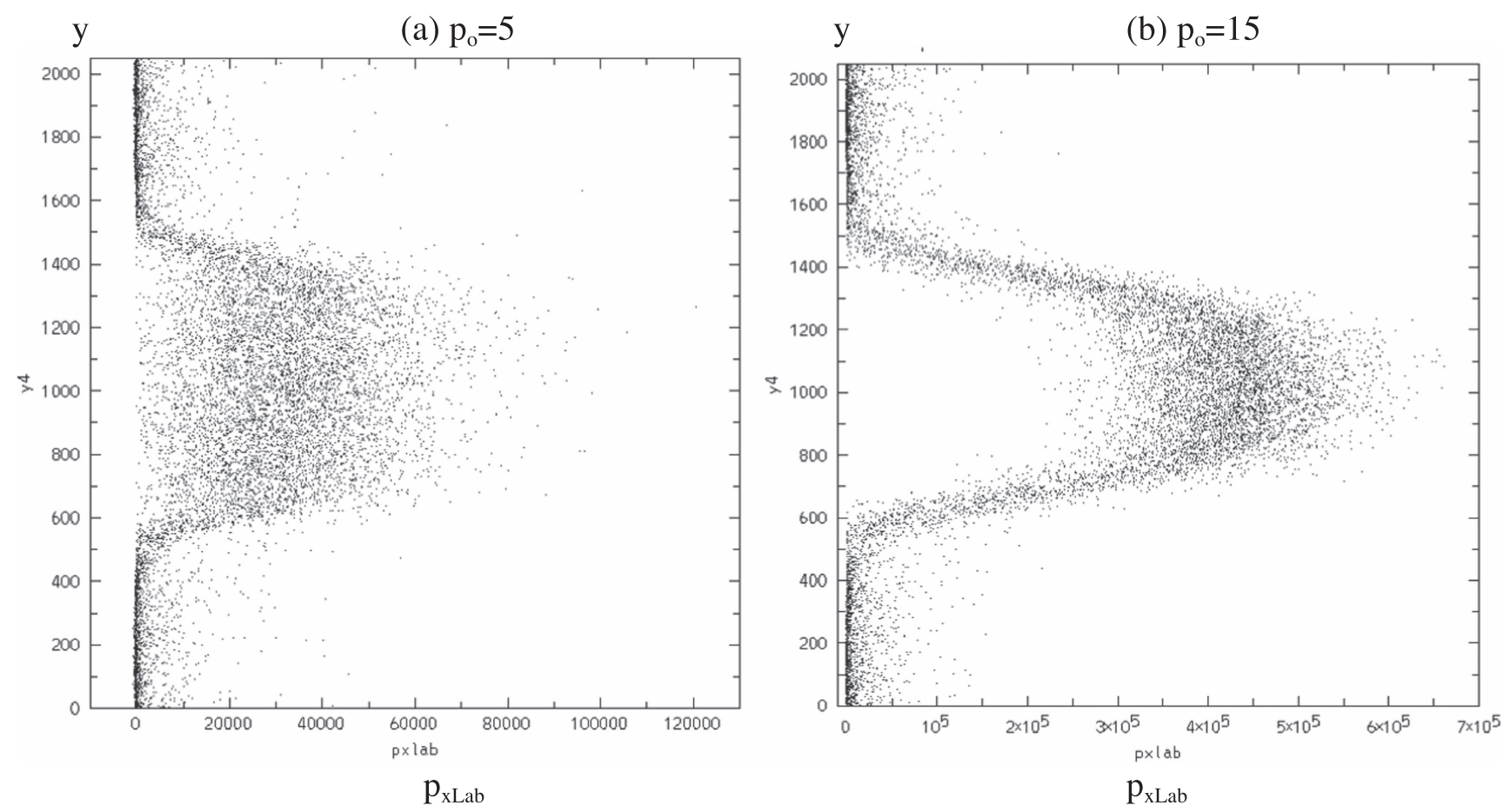

Figure 7. Phase plot ( $\boldsymbol{y}$ vs. $\boldsymbol{p}_{x \mathrm{Lab}}$ ) of spine electrons for the $p_{\mathrm{o}}=5$ case (a) compared to that of the $p_{\mathrm{o}}=15$ case (b) at $t \omega_{\mathrm{e}}=8000$, after Lorentz boosting $\boldsymbol{p}_{x}$ to the "laboratory frame" in which the sheath is initially at rest. By this time, some of the spine electrons have diffused into the sheath region and are decelerated, forming the low-energy population at the left. The central high-energy population corresponds to electrons remaining in the spine. Note that electrons are more concentrated at the highest energy for the $p_{\mathrm{o}}=15$ case. 

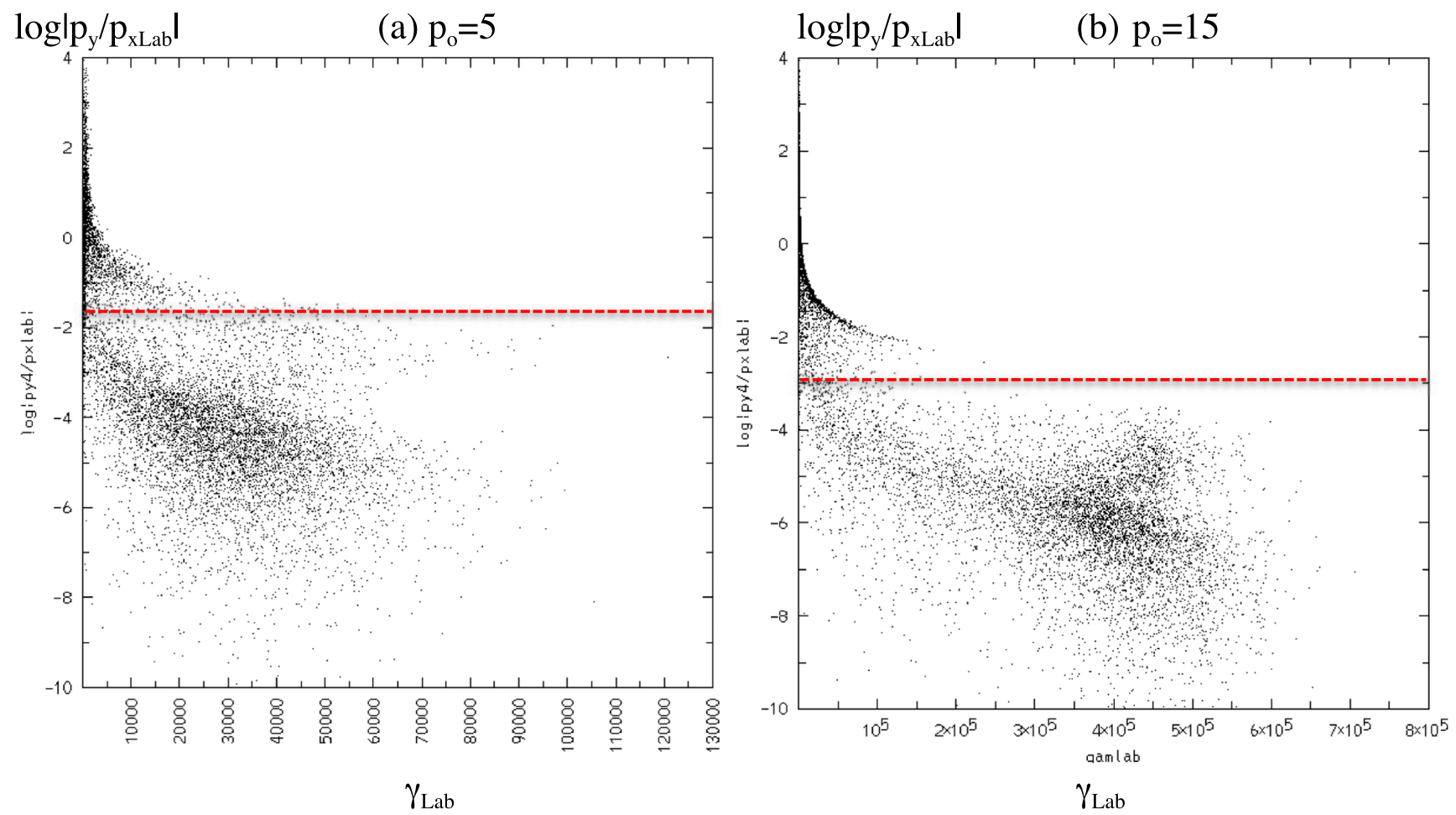

Figure 8. Distribution of the tangent of the "beam angle" $\left(=\left|\boldsymbol{p}_{y} / \boldsymbol{p}_{x \mathrm{Lab}}\right|\right)$ of spine electrons vs. Lorentz factor $\gamma_{\mathrm{Lab}}$ in the "laboratory frame" at $t \omega_{\mathrm{e}}=8000$ for (a) $p_{\mathrm{o}}=5$ and (b) $p_{\mathrm{o}}=15$. We see that in both cases all of the high-energy spine electrons (i.e., those that did not cross over to the sheath and get decelerated) have beam angles much smaller than $1 / \Gamma$ (red dashed lines). In both cases, there exists an exponential anti-correlation between beam angle and electron energy, which may be tested with future observations.

\section{ORCID iDs}

Wen Fu (i) https://orcid.org/0000-0003-2646-0577

Markus Böttcher (i) https://orcid.org/0000-0002-8434-5692

\section{References}

Abdo, A. A., Ackermannn, M., Agudo, I., et al. 2010, ApJ, 716, 30 Alves, E. P., Griesmayer, T., Fonseca, R. A., \& Silva, L. O. 2014, NJPh, 16, 035007

Alves, E. P., Griesmayer, T., Fonseca, R. A., \& Silva, L. O. 2015, PhRvE, 92, 021101

Alves, E. P., Grismayer, T., Martins, S. F., et al. 2012, ApJL, 746, L14

Birdsall, C., \& Langdon, A. B. 1991, Plasma Physics via Computer Simulation (Bristol: Institute of Physics Publishing)

Boettcher, M. 2007, Ap\&SS, 309, 95

Boettcher, M., Reimer, A., Sweeney, K., \& Prakash, A. 2013, ApJ, 768, 54

Bonnoli, G., Tavecchio, F., Ghisellini, G., \& Sbarrato, T. 2015, MNRAS, 451, 611

Chandrasekhar, S. 1981, Hydrodynamic and Hydromagnetic Stability (New York: Dover)

Ghisellini, G., Tavecchio, F., \& Chiaberge, M. 2005, A\&A, 432, 401

Ghisellini, G., Tavecchio, F., Foschini, L., et al. 2010, MNRAS, 402, 497

Giroletti, M., Giovannini, G., Feretti, L., Cotton, W. D., \& Edwards, P. G. 2004, ApJ, 600, 127

Godfrey, B. 1974, JCoPh, 15, 504

Godfrey, B. 1975, JCoPh, 19, 58
Godfrey, B., \& Langdon, B. 1976, JCoPh, 20, 251

Grismayer, T., Alves, E. P., Fonseca, R. A., \& Silva, L. O. 2013, PhRvL, 111, 015005

Hovatta, T., Valtaoja, E., Tornikoski, M., et al. 2009, A\&A, 494, 527

Jorstad, S., Svetlana, G., Marscher, A., et al. 2005, AJ, 130, 1418

Langdon, A. B., \& Lasinski, B. 1976, in Methods in Computational Physics (New York: Academic Press), 327

Liang, E., Boettcher, M., \& Smith, I. 2013a, ApJL, 766, L19

Liang, E., Fu, W., \& Boettcher, M. 2017, ApJ, 847, 90

Liang, E., Fu, W., Boettcher, M., Smith, I., \& Roustazadeh, P. 2013b, ApJL, 779, L27

Lyutikov, M., \& Lister, M. 2010, ApJ, 722, 197

Meliani, Z., \& Keppens, R. 2007, A\&A, 475, 785

Meliani, Z., \& Keppens, R. 2009, ApJ, 705, 1594

Meszaros, P. 2002, ARA\&A, 40, 137

Mizuno, Y., Hardee, P., \& Nishikawa, K.-I. 2007, ApJ, 662, 835

Nagai, H., Haga, T., Giovannini, G., et al. 2014, ApJ, 785, 53

Nishikawa, K., Frederiksen, J., Nordlund, A., et al. 2016, ApJ, 820, 94

Nishikawa, K., Hardee, P., Zhang, B., et al. 2013, AnGeo, 31, 1535

Nishikawa, K., Hardee, P., Dutan, I., et al. 2014, ApJ, 793, 60

Piran, T. 2004, RvMP, 76, 1143

Preece, R. D., Briggs, M. S., Mallozzi, R. S., et al. 1998, ApJL, 506, L23

Rybicki, G., \& Lightman, A. 1979, Radiative Processes in Astrophysics (San Francisco, CA: Freeman)

Sironi, L., \& Spitkovsky, A. 2009, ApJL, 707, L92

Tavecchio, F., \& Ghsiellini, G. 2008, MNRAS, 385, L98

Zhang, W., MacFadyen, A., \& Wang, P. 2009, ApJL, 692, L40 